\title{
Predicting radio emission from the newborn hot Jupiter V830 Tauri $b$ and its host star
}

\author{
A. A. Vidotto ${ }^{1}$ and J.-F. Donati ${ }^{2,3}$ \\ ${ }^{1}$ School of Physics, Trinity College Dublin, University of Dublin, Ireland \\ e-mail: Aline.Vidotto@tcd.ie \\ ${ }^{2}$ Université de Toulouse, UPS-OMP, IRAP, 14 avenue É. Belin, 31400 Toulouse, France \\ 3 CNRS, IRAP/UMR 5277, 14 avenue É. Belin, 31400 Toulouse, France
}

Received 12 September 2016 / Accepted 4 March 2017

\begin{abstract}
Magnetised exoplanets are expected to emit at radio frequencies analogously to the radio auroral emission of Earth and Jupiter. Here, we predict the radio emission from V830 Tau b, the youngest (2 Myr) detected exoplanet to date. We model the wind of its host star using three-dimensional magnetohydrodynamics simulations that take into account the reconstructed stellar surface magnetic field. Our simulations allow us to constrain the local conditions of the environment surrounding V830 Tau b that we use to then compute its radio emission. We estimate average radio flux densities of 6 to $24 \mathrm{mJy}$, depending on the assumption of the radius of the planet (one or two Jupiter radii). These radio fluxes are not constant along one planetary orbit, and present peaks that are up to twice the average values. We show here that these fluxes are weakly dependent (a factor of 1.8) on the assumed polar planetary magnetic field $(10$ to $100 \mathrm{G})$, opposed to the maximum frequency of the emission, which ranges from 18 to $240 \mathrm{MHz}$. We also estimate the thermal radio emission from the stellar wind. By comparing our results with the Karl G. Jansky Very Large Array and the Very Long Baseline Array observations of the system, we constrain the stellar mass-loss rate to be $\lesssim 3 \times 10^{-9} M_{\odot} \mathrm{yr}^{-1}$, with likely values between $\sim 10^{-12}$ and $10^{-10} M_{\odot} \mathrm{yr}^{-1}$. With these values, we estimate that the frequency-dependent extension of the radio-emitting wind is around $\sim 3$ to 30 stellar radii $\left(R_{\star}\right)$ for frequencies in the range of 275 to $50 \mathrm{MHz}$, implying that V830 Tau b, at an orbital distance of $6.1 R_{\star}$, could be embedded in the regions of the host star's wind that are optically thick to radio wavelengths, but not deeply so. We also note that planetary emission can only propagate in the stellar wind plasma if the frequency of the cyclotron emission exceeds the stellar wind plasma frequency. In other words, we find that for planetary radio emission to propagate through the host star wind, planetary magnetic field strengths larger than $\sim 1.3$ to $13 \mathrm{G}$ are required. Since our radio emission computations are based on analogies with solar system planets, we caution that our computations should be considered as estimates. Nevertheless, the V830 Tau system is a very interesting system for conducting radio observations from both the perspective of radio emission from the planet as well as from the host star's wind.
\end{abstract}

Key words. stars: low-mass - stars: winds, outflows - planet-star interactions - planets and satellites: magnetic fields

\section{Introduction}

Detecting radio emission from exoplanets would revolutionise the area of exoplanetary studies. First, it would open up a new avenue for the direct detection of exoplanets (Farrell et al. 1999). Second, it would allow us to measure exoplanetary magnetic fields, which so far have only been elusively probed (Shkolnik et al. 2008; Vidotto et al. 2010a; Kislyakova et al. 2014). Exoplanetary magnetic fields can reveal information about a planet's interior structure and dynamics (e.g. whether it has a convecting, electrically conducting interior) and are also believed to play an important role in conditions for habitability, by protecting the planet's surface from energetic cosmic particles, protecting its atmosphere from violent chemical changes, and potentially helping atmospheric retention (Lundin et al. 2007; Grießmeier et al. 2016).

The magnetised planets in the solar system generate radio emission through the electron cyclotron maser instability. The power of this emission is related to the kinetic and/or magnetic powers of the incident solar wind over several orders of magnitude (Farrell et al. 1999; Zarka et al. 2001). Although the physics of this relation are still not well understood, this "radiometric Bode's relation" indicates that planetary radio emission is somehow powered by the interaction between the planetary magnetic field and the solar wind. In analogy to the solar system, it has been suggested that exoplanets may also produce radio emission due to their interaction with the winds of their host stars (Farrell et al. 1999) ${ }^{1}$.

Calculations based on the solar-system analogy suggest that radio emission from close-in exoplanets should be several orders of magnitude larger than the largest radio emitter of the solar system, Jupiter (e.g., Zarka et al. 2001; Lazio et al. 2004; Grießmeier et al. 2005; Zarka 2007; Vidotto et al. 2010b; Fares et al. 2010; Hess \& Zarka 2011). This is because, at the short orbital distances of close-in planets, the local power of the host star wind is significantly higher than, for example, the local power of the solar wind dissipated at the distances of the planets in our solar system. These and many other theoretical predictions

\footnotetext{
1 In particular, Zarka (2010) and Zarka et al. (2015) found that the scaling between the magnetic power of the incident stellar wind on the magnetospheric cross-section and the radio power apply also to any plasma flow-obstacle interaction, including interactions between Io and Ganymede with Jupiter and the magnetised binary system V711 Tau. This generalised radio-magnetic Bode's law covers about 13 orders of magnitude in stellar wind magnetic power and radio power.
} 
have motivated a good number of observational searches of exoplanetary radio emission, yielding mostly negative results (e.g. Bastian et al. 2000; Ryabov et al. 2004; Lazio \& Farrell 2007; Hallinan et al. 2013; but see also Lecavelier des Etangs et al. 2013; Sirothia et al. 2014, who found ambiguous hints of the existence of exoplanetary radio emissions). There are several reasons for the non-detections, such as a lack of exoplanetary magnetism, a mismatch of the frequency search, and/or a low sensitivity of the present-day instruments (Bastian et al. 2000; Grießmeier et al. 2011).

One might overcome the issue of instrumental sensitivity by turning to exoplanets that, not only orbit closer to their stars, but that orbit stars that have winds that are more powerful than solar-type winds, and whose magnetic fields are also stronger. Stars that are more active than the Sun are indeed expected to host more powerful winds (Wood 2004; See et al. 2014; Johnstone et al. 2015; do Nascimento et al. 2016). In particular, stars in the pre-main sequence with close-in planets would be ideal targets to search for planetary radio emission (Grießmeier et al. 2005; Vidotto et al. 2010b). With the recent detection of the first hot giants orbiting stars younger than 10-20 Myr (V830 Tau b, Donati et al. 2016; and K2-33b, David et al. 2016 TAP26b; Yu et al. 2017), now is an excellent timing for testing theoretical expectations of exoplanetary radio emission. In this paper, we compute the expected radio emission from the newborn hot Jupiter V830 Tau b.

With the spectropolarimetric monitoring of V830 Tau, Donati et al. (2016) were able to extract the planetary signature of V830 Tau b, a 2 Myr-old hot Jupiter orbiting at $0.057 \mathrm{au}$, while, at the same time, reconstructing the large-scale magnetic field topology of the host star. It is precisely the latter measurements that we use in this paper to derive the wind properties of the host star (Sect. 2). With that, we are then able to derive the expected radio emission of V830 Tau $b$, starting from the assumption that V830 Tau b is magnetised (Sect. 3). Given the high densities of the wind of young active stars, these winds can become optically thick to free-free radiation at radio wavelengths. We thus investigate in which circumstances the planet would be embedded in the radio-emitting region of the stellar wind and whether planetary radio emission would be able to propagate through the stellar wind plasma, given that the planetary cyclotron frequency of emission should exceed the plasma frequency of the stellar wind (Sect. 4). For physicallyreasonable conditions, we show that planetary radio emission from V830 Tau b is expected to peak at $12 \mathrm{mJy}$, presenting therefore a high potential of detection with the Low-Frequency Array (LOFAR) the upgraded Ukrainian T-shaped Radio telescope (UTR-2) and Giant Metrewave Radio Telescope (GMRT, see Sect. 5). Interestingly, its host star has been detected using the Karl G. Jansky Very Large Array (VLA) at 4.5 and $7.5 \mathrm{GHz}$ and with the Very Long Baseline Array (VLBA) at $8.4 \mathrm{GHz}$, becoming the first exoplanet host with detected radio emission (Bower et al. 2016).

\section{Stellar wind modelling}

We model the wind of V830 Tau by means of three-dimensional (3D) magnetohydrodynamics (MHD) simulations, similarly to Vidotto et al. $(2012,2015)$. We refer the reader to these papers for more details of the model, which are summarised next. We use the 3D MHD numerical code BATS-R-US (Powell et al. 1999; Tóth et al. 2012), modified as in Vidotto et al. (2012). BATS-R-US solves the set of ideal MHD equations for the mass density, velocity, magnetic field, and gas pressure. We assume the wind is polytropic with a polytropic index $\gamma=1.15$ and a fully ionised hydrogen wind.

For the physical characteristics of the star, we use a rotation period of 2.741 days, mass of $1 M_{\odot}$, and radius of $R_{\star}=$ $2 R_{\odot}$. The radial part of the stellar magnetic field, anchored at the wind base, is constrained from observations collected in November-December 2015 (Donati et al. 2016). Initially, the field is considered to be in its lowest (potential) state, but as the simulation evolves in time the wind particles self-consistently interact with the magnetic field lines (and vice-versa), removing the field from its initial potential state. At the wind base, we adopt a temperature of $10^{6} \mathrm{~K}$ and a number density of $n_{0}=10^{12} \mathrm{~cm}^{-3}$, which are free parameters of our model. The star rotates as a solid body with a rotation axis along the $z$-axis. Our grid is the same as described in Vidotto et al. (2014b). The resultant wind solution, obtained self-consistently, is found when the system reaches steady state in the reference frame corotating with the star. For the parameters we adopted, we obtain a wind mass-loss rate of $\dot{M} \sim 3 \times 10^{-9} M_{\odot} \mathrm{yr}^{-1}$. This mass-loss rate is an upper limit for the case V830 Tau, as we will demonstrate in Sect. 4.

Figure 1 illustrates the output of the wind simulations of V830 Tau. We overplot the (assumed equatorial and circular) orbital radius of V830 Tau b (black circle) and a cut of the Alfvén surface $S_{\mathrm{A}}$ at the equatorial plane ( $x y$ plane). For the parameters we adopted, the planet's orbit lies almost completely inside $S_{\mathrm{A}}$. The Alfvén surface is expected to expand for lower values of $n_{0}$ (Vidotto et al. 2009). This indicates that V830 Tau b is truly orbiting in the sub-Alfvénic regime, if not at all times, at least during most of its orbit. The Alfvén surface represents the boundary between a magnetically-dominated stellar wind (interior to $S_{\mathrm{A}}$ ) and a region that is dominated by the wind inertia. The Alfvén surface is relevant for computing angular momentum losses (Weber \& Davis 1967) and indicates the types of star-planet interactions. Planets orbiting in the sub-Alfvénic regime can be directly connected to the magnetic field of the star and perturbations can travel to/from the star (e.g. Preusse et al. 2005; Saur et al. 2013; Strugarek et al. 2015). In this case, the planetary magnetosphere is embedded in that of the star and it is believed that there is continuous reconnection of the planet's and stellar magnetic fields, similar to the Ganymede-Jupiter system. When the planet orbits outside $S_{\mathrm{A}}$ (in the super-Alfvénic regime), as in the case of the Earth, the magnetospheric cavity surrounding the planet deflects the stellar wind particles. We estimate the size of the magnetosphere of V830 Tau b in the next section.

\section{Exoplanetary radio emission}

To calculate the radio emission of V830 Tau b, we use the radiometric Bode's law for the solar system, in which the planetary radio power $P_{\text {radio }}$ can be decomposed into a power released from the dissipation of kinetic energy of the stellar wind $P_{\mathrm{k}}$ and/or a power released from the dissipation of magnetic energy of the wind $P_{B}$ (e.g. Farrell et al. 1999; Zarka et al. 2001)

$P_{\text {radio }}=\eta_{\mathrm{k}} P_{\mathrm{k}} \quad$ or $\quad P_{\text {radio }}=\eta_{B} P_{B}$,

where $\eta_{\mathrm{k}}$ and $\eta_{B}$ are efficiency ratios. In the solar system, $\eta_{\mathrm{k}}=$ $1 \times 10^{-5}$ and $\eta_{B}=2 \times 10^{-3}$ (Zarka 2007); we assume the same efficiency ratios here. In the past, it has been argued that it was not possible to decide which incident power actually drives the radio power observed from the magnetic planets of the solar system (Zarka 2007). Recently, however, Zarka (2010) argued that 


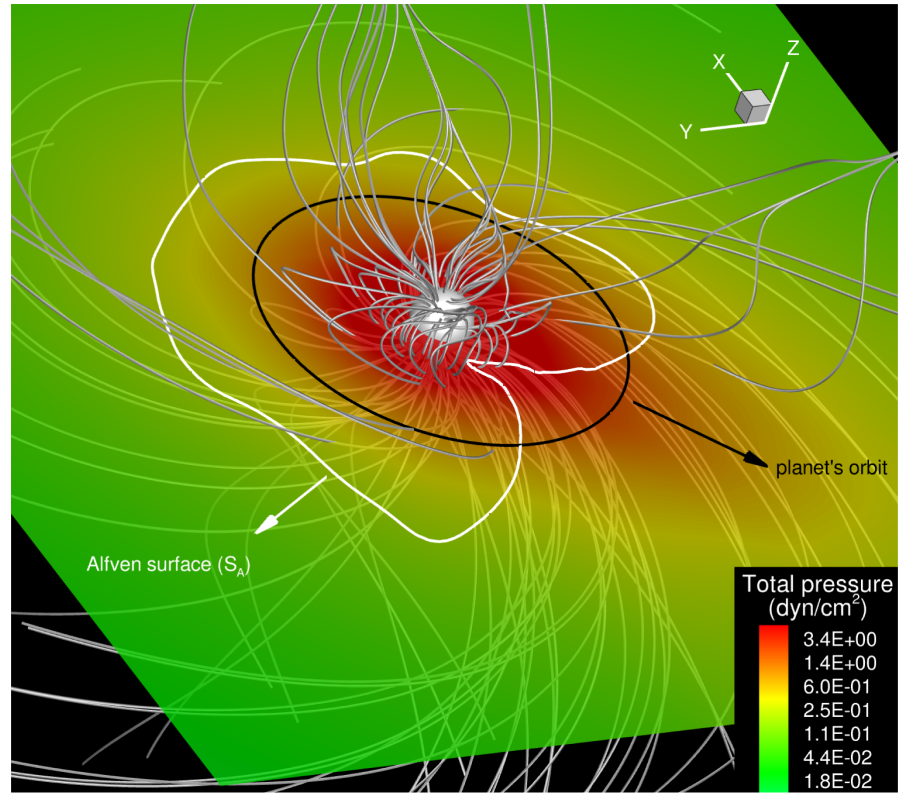

Fig. 1. Simulated wind of V830 Tau. Thin grey lines represent the magnetic field of V830 Tau that is embedded in the wind. The circle depicts the orbital radius of V830 Tau $\mathrm{b}$, assumed to lie in the equatorial plane of the star ( $x y$ plane). The white line represents a cut of the Alfvén surface at the equatorial plane, while the colour shows the total wind pressure.

the magnetic field is likely to be a determinant for extracting part of the flow power and converting it to energetic particles (see e.g. Jardine \& Cameron 2008; Nichols \& Milan 2016, for a theoretical basis of this empirical relation). Here, for completeness, we compute the radio powers coming from both kinetic and magnetic energies. As we will see, the latter is much larger than the former and, following the arguments in Zarka (2010), is the preferred method for estimating radio fluxes.

The dissipated kinetic and magnetic powers of the impacting wind on the planet are approximated as, respectively (Appendix A),

$P_{\mathrm{k}} \simeq \rho(\Delta u)^{3} \pi r_{\mathrm{M}}^{2}$,

$P_{B} \simeq \frac{B_{\perp}^{2}}{4 \pi}(\Delta u) \pi r_{\mathrm{M}}^{2}$,

where $B_{\perp}$ is the magnetic field component perpendicular to $\Delta u$. Here, $|\Delta \boldsymbol{u}|=\left|\boldsymbol{u}-\boldsymbol{v}_{\mathrm{K}}\right|$ is the relative velocity between the wind and the Keplerian velocity $\left(v_{\mathrm{K}}\right)$ of the planet, and $\rho, p$, and $B$ are the local density, pressure, and magnetic field intensity of the stellar wind. Neglecting planet thermal pressure, the size of the planet's magnetopause $r_{\mathrm{M}}$ can be estimated by pressure balance between the stellar wind total pressure and the planet's magnetic pressure: $p_{\text {tot }}=B_{\mathrm{p}}^{2}\left(r_{\mathrm{M}}\right) /(8 \pi)$, where $p_{\text {tot }}=\rho \Delta u^{2}+\frac{B^{2}}{8 \pi}+p$ is the sum of the ram, magnetic, and thermal pressures of the wind; $B_{\mathrm{p}}\left(r_{\mathrm{M}}\right)$ is the intensity of the planet's magnetic field at the nose of the magnetopause. For a dipolar planetary magnetic field: $B_{\mathrm{p}}\left(r_{\mathrm{M}}\right)=\frac{1}{2} B_{\mathrm{p}}\left(R_{\mathrm{p}} / R\right)^{3}$, where $R_{\mathrm{p}}$ is the planetary radius, $R$ is the radial coordinate centred at the planet, and $B_{\mathrm{p}}$ is the polar magnetic field intensity (at the equator, the intensity is $\frac{1}{2} B_{\mathrm{p}}$ ). Assuming the dipole is aligned with the planetary orbital spin axis, the magnetospheric radius (where $R=r_{\mathrm{M}}$ ) is

$\frac{r_{\mathrm{M}}}{R_{\mathrm{p}}}=2^{1 / 3}\left[\frac{\left(B_{\mathrm{p}} / 2\right)^{2} / 8 \pi}{\rho \Delta u^{2}+p+B^{2} / 8 \pi}\right]^{1 / 6}$.

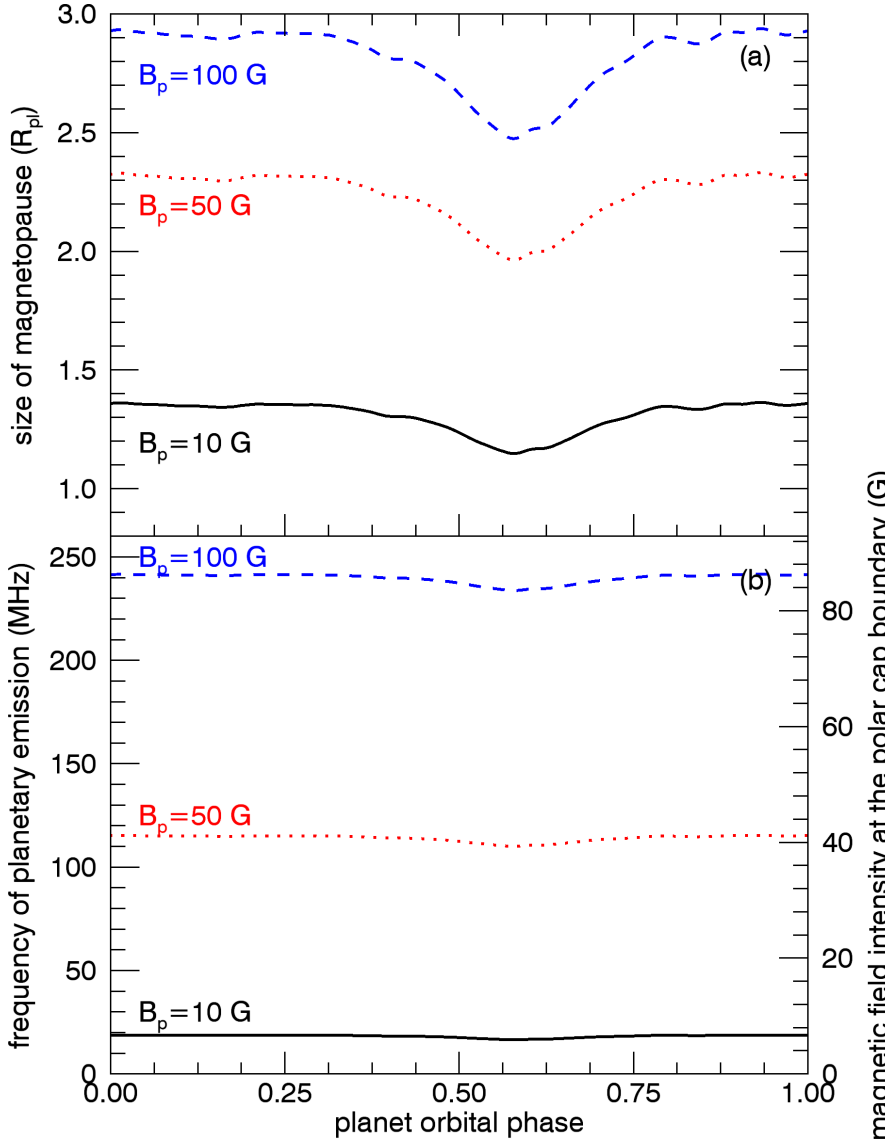

Fig. 2. a) Estimated sizes of the planetary magnetospheres along a planetary year assuming three different values of the polar planetary magnetic field: 10, 50, and $100 \mathrm{G}$. b) Predicted maximum frequency of the radio emission of V830 Tau $b$. This frequency is calculated at the border of the polar-cap boundary, which is located at different colatitudes depending on the size of the planet magnetosphere (cf., Eq. (A.1)). The magnetic field intensity at this position in shown in the right axis.

Figure 2a shows the stand-off distance of V830 Tau b's magnetopause calculated using Eq. (4) as the planet orbits around the $\operatorname{star}^{2}$. We assume three different values of $B_{\mathrm{p}}: 10,50$, and $100 \mathrm{G}$. For comparison, we note that the maximum intensity of Jupiter's magnetic field is $14.3 \mathrm{G}$ (Bagenal 1992). For $B_{\mathrm{p}}=10 \mathrm{G}$, the average magnetospheric radius is $\sim 1.31 R_{\mathrm{p}}$ (i.e. very close to the planet surface), while in the second and third cases, $r_{\mathrm{M}} \sim 2.2 R_{\mathrm{p}}$ and $2.8 R_{\mathrm{p}}$, respectively. If the wind densities (and therefore mass-loss rates) were to decrease by a factor of 100 , the magnetospheric sizes would increase by about $20 \%$ of the values presented in Fig. 2a (in such a case, the planet's orbit would lie completely within the Alfvén surface). This shows that, even in the case of a "high" planetary magnetic field and low wind densities, the magnetosphere of V830 Tau b is expected to be quite small, due to the harsh conditions of the external environment.

The radio flux is related to the radio power as

$\phi_{\text {radio }}=\frac{P_{\text {radio }}}{d^{2} \omega \Delta f}$,

where $\omega$ is the solid angle of the (hollow) cone of emission, $d$ is the distance to the system (147 pc in the case of V830 Tau), and $\Delta f$ is the emission bandwidth, approximately the cyclotron frequency (Grießmeier et al. 2007). In Appendix A, we show that

2 The right hand side of Eq. (4) is often multiplied by a correction factor $2^{1 / 3}$ used to account for the effects of currents (e.g. Cravens 2004). 
the radio flux density due to the dissipated wind kinetic and magnetic powers simplify to

$\phi_{\text {radio,kin }}=\eta_{\mathrm{k}}^{\prime} \frac{R_{\mathrm{p}}^{2}}{d^{2}} \frac{\rho(\Delta u)^{3}}{p_{\text {tot }}^{1 / 2}} f\left(\alpha_{0}\right)$,

$\phi_{\text {radio,mag }}=\eta_{B}^{\prime} \frac{R_{\mathrm{p}}^{2}}{d^{2}} \frac{B_{\perp}^{2}(\Delta u)}{p_{\text {tot }}^{1 / 2}} f\left(\alpha_{0}\right)$,

respectively. Here $\eta_{\mathrm{k}}^{\prime}$ and $\eta_{B}^{\prime}$ are constants and are related to $\eta_{\mathrm{k}}$ and $\eta_{B}$ following Eqs. (A.13) and (A.19). The planetary magnetic field dependence is hidden in the function $f\left(\alpha_{0}\right)$, which varies between 0 and 3.3 for any planetary magnetic field intensity (Fig. A.1). In particular, we assume that the beaming angle of the radio emission occurs at a ring with colatitude $\alpha_{0}$ and thickness $\delta \alpha=17.5^{\circ}$ (Zarka et al. 2004). Using the values for $\eta_{\mathrm{k}}$ and $\eta_{B}$ from the solar system, we have $\eta_{\mathrm{k}}^{\prime} \simeq$ $1.8 \times 10^{-13}$ [cgs units] and $\eta_{B}^{\prime} \simeq 2.8 \times 10^{-12}$ [cgs units]. From Eqs. (6), (7), (A.13), and (A.19), we have that

$$
\frac{\phi_{\text {radio,kin }}}{\phi_{\text {radio,mag }}}=\frac{\eta_{\mathrm{k}}}{\eta_{B}(4 \pi)^{2}} \frac{\rho(\Delta u)^{2} / 2}{B_{\perp}^{2} / 8 \pi}=\frac{1}{3200 \pi^{2}} \frac{\rho(\Delta u)^{2} / 2}{B_{\perp}^{2} / 8 \pi} .
$$

Equation (8) shows that the flux released in the dissipation of Poynting flux becomes dominant when the kinetic energy $\left(\rho(\Delta u)^{2} / 2\right)$ of the impacting wind is smaller than $3200 \pi^{2}$ times the magnetic energy $\left(B_{\perp}^{2} / 8 \pi\right)$. This is the case of the V830 Tau system, due to the intense stellar magnetism.

From Eqs. (6) and (7), we see that the radio fluxes are functions of the angular size of the planet $\left(R_{\mathrm{p}} / d\right)$ and the properties of the stellar wind surrounding the planet. We also note that radio fluxes depend relatively weakly on the magnetic intensity of the planetary dipolar field. This dependence is hidden in $f\left(\alpha_{0}\right)$ (cf. Appendix A). For example, for polar magnetic fields of 10, 50 , and $100 \mathrm{G}$, respectively, the (northern) polar-cap boundaries are located at colatitudes $\alpha_{0}$ of $61^{\circ}, 42^{\circ}$, and $37^{\circ}$, respectively. For these values of $\alpha_{0}, f\left(\alpha_{0}\right)$ is $2.2,1.4$, and 1.2, respectively. Therefore, as radio fluxes are proportional to $f\left(\alpha_{0}\right)$, a change of field intensities from 10 to $100 \mathrm{G}$ results in a rather small change (a factor of 1.8) in the computed radio fluxes. This is good news, given that exoplanetary magnetism remains observationally elusive (cf. Sect. 1).

Figure 3 shows the predicted radio flux densities along a planetary year assuming dissipation of kinetic (top) and magnetic (bottom) stellar wind powers. We found that radio emission arising from the dissipation of wind magnetic power is considerably larger ( $\sim 30$ times) than from kinetic power and this difference could become even larger should a lower stellar mass-loss rate be adopted. This is due to the kinetic-to-magnetic ratio being smaller than $3200 \pi^{2}$ (cf. Eq. (8)). In Appendix B, we show that our radio predictions for $\mathrm{V} 830$ Tau $\mathrm{b}$ are robust against a reduction of stellar wind densities and mass-loss rates, given the high magnetism of V830 Tau. We assume here that the planet has a radius of 1 to $2 R_{\text {jup }}$, but we note that, although the mass of V830 Tau $\mathrm{b}$ has been constrained by observations to be about $0.7 M_{\text {jup }}$ (Donati et al. 2016), no constraints on its radius currently exist. Since radio fluxes are proportional to $R_{\mathrm{p}}^{2}$, the fluxes calculated for $R_{\mathrm{p}}=2 R_{\text {jup }}$ are four times larger than for the calculations with $R_{\mathrm{p}}=R_{\text {jup }}$. For the case of $R_{\mathrm{p}}=R_{\text {jup }}$, the estimated magnetic radio fluxes are on average $\sim 6 \mathrm{mJy}$, with peaks at $11 \mathrm{mJy}$. This can be considered as a conservative estimate. Due to its youth and the close distance to the host star, it is likely that the radius of V830 Tau b is larger than $1 R_{\text {jup }}$, since V830 Tau b is likely to be still in the contracting phase (e.g. K2$29 \mathrm{~b}$, which has a similar mass, but orbits an older, $450 \mathrm{Myr}$-old

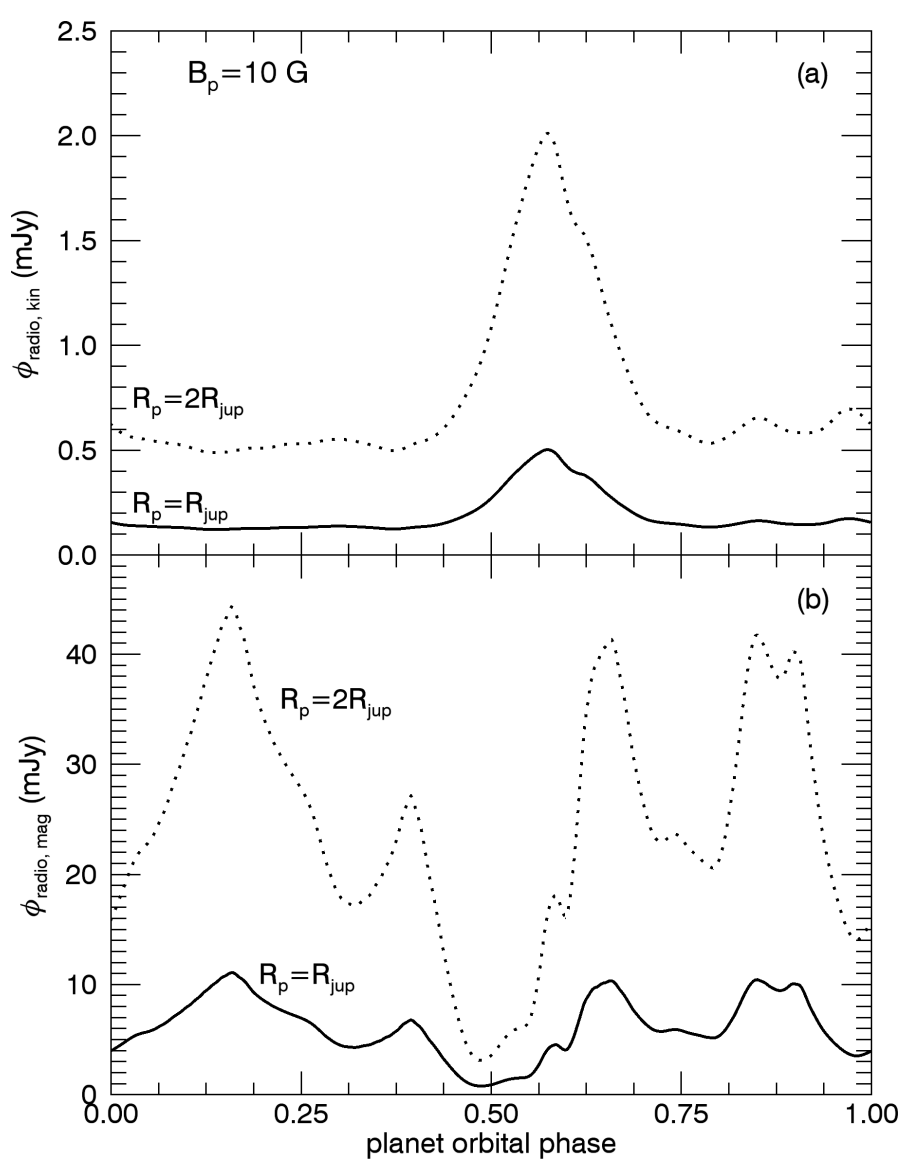

Fig. 3. Predicted radio flux density along a planetary year assuming dissipation of kinetic (top) and magnetic (bottom) stellar wind powers onto the magnetospheric cross section of the exoplanet. At the low frequency range, values of a few mJy are potentially observable with LOFAR, the upgraded UTR-2 and GMRT.

star, has a radius of $1.19 R_{\text {jup }}$, Santerne et al. 2016). For the case of $R_{\mathrm{p}}=2 R_{\text {jup }}$, the estimated magnetic radio fluxes are on average $\sim 24 \mathrm{mJy}$, with peaks at $44 \mathrm{mJy}$.

We adopted in Fig. 3 a dipolar magnetic field whose polar intensity is $10 \mathrm{G}$, but again note that an increase in magnetic field intensity by a factor of 10 would result in a change on the predicted radio flux by a factor of 1.8. The planetary magnetic field has, however, a strong influence on the frequency of emission. Since this is cyclotron emission, by measuring its frequency one is able to derive the intensity of the planetary magnetic field (cf. Eq. (A.3), see Hess \& Zarka 2011, for a suggestion on how to conduct this estimation). Figure $2 \mathrm{~b}$ shows the predicted maximum emission frequency, considering the same polar planetary magnetic fields as in Fig. 2a. This frequency is calculated at the border of the polar-cap boundary, which is located at different colatitudes depending on the size of the planet magnetosphere (cf. Eq. (A.1), Vidotto et al. 2011). The frequencies are 18, 114, and $240 \mathrm{MHz}$ for polar magnetic fields of 10,50, and $100 \mathrm{G}$, respectively. The polar-cap boundaries extend down to latitudes of $\pm 29^{\circ}, \pm 48^{\circ}$, and $\pm 53^{\circ}$, respectively. The magnetic field intensity at these latitudes is shown in the right axis of Fig. 2b. For comparison, at the Earth, the size of the polar cap is about 17 to $20^{\circ}$ (Milan et al. 2009), that is, extending to latitudes of \pm 70 to $\pm 73^{\circ}$, showing once more the extreme case of the magnetosphere of V830 Tau b. 


\section{Stellar versus exoplanetary radio emissions}

If V830 Tau b is indeed a magnetised planet, it can emit at radio wavelengths due to a physical process called electron cyclotron maser instability (Sect. 3). However, V830 Tau b may not be the only radio emitter in this exoplanetary system. The hot plasma from the stellar wind can also emit at radio wavelengths, although through a different physical process. Here, we estimate radio emission from the host star's wind.

\subsection{Radio emission from the stellar wind}

A thermal ionised plasma emits bremsstrahlung (free-free) radiation across the electromagnetic spectrum. In the case of stellar winds, free-free radio emission is more intense in the innermost regions of the stellar wind, where the densities are higher (Panagia \& Felli 1975; Wright \& Barlow 1975; Lim \& White 1996; Guedel 2002). For large enough densities, these regions can become optically thick to radio wavelengths and, if a planet is embedded in this region, then it is possible that most of the planetary radio emission gets absorbed and does not escape.

A simple way to calculate the radio emission of stellar winds was presented in Panagia \& Felli (1975), who assume that the wind is spherically symmetric and isothermal with temperature $T$. These hypotheses are, however, not true in our simulations: the asymmetric distribution of surface magnetic fields generates an asymmetric stellar wind (Vidotto et al. 2014b). We also adopt a polytropic wind model, such that the wind temperature is not constant. To take into account the asymmetries of the stellar wind, a radiative transfer calculation performed on each cell of the numerical grid and integrated along the line-of-sight would provide more accurate predictions for the radio-emitting wind. This detailed study will be done in a future work. In the present paper, we compute the free-free emission of the inner regions of the wind of V830 Tau using the model developed by Panagia \& Felli (1975) coupled to our simulation results. We caution that these computations should be considered as estimates of the radio emission.

The stellar wind radio flux density at a frequency $v$ is given by (Panagia \& Felli 1975)

$$
\begin{aligned}
S_{v}= & 10^{-29} A(\alpha) R_{\star}^{2}\left[5.624 \times 10^{-28} I(\alpha) n_{0}^{2} R_{\star}\right]^{\frac{2}{2 \alpha-1}} \\
& \times\left(\frac{v}{10 \mathrm{GHz}}\right)^{\frac{-4.2}{2 \alpha-1}+2}\left(\frac{T}{10^{4} \mathrm{~K}}\right)^{\frac{-2.7}{2 \alpha-1}+1}\left(\frac{d}{1 \mathrm{kpc}}\right)^{-2} \mathrm{mJy},
\end{aligned}
$$

where the functions $I(\alpha)$ and $A(\alpha)$ are given by

$$
\begin{aligned}
& I(\alpha)=\int_{0}^{\pi / 2}(\sin \theta)^{2(\alpha-1)} \mathrm{d} \theta, \\
& A(\alpha)=1+2 \sum_{j=1}^{\infty}(-1)^{j+1} \frac{\tau_{\mathrm{c}}^{j}}{j ! j(2 \alpha-1)-2},
\end{aligned}
$$

and $\tau_{\mathrm{c}}=3$. The wind density is assumed to decay as a power-law with exponent $\alpha$

$n=n_{0}\left(\frac{R_{\star}}{r}\right)^{-\alpha}$,

with $n_{0}$ being the wind base density. The distance $R_{v}$ within which half of the emission $S_{v}$ is produced is

$$
\frac{R_{v}}{R_{\star}}=\left[4.23 \times 10^{-27} I(\alpha) n_{0}^{2} R_{\star}\right]^{\frac{1}{2 \alpha-1}}\left(\frac{v}{10 \mathrm{GHz}}\right)^{\frac{-2.1}{2 \alpha-1}}\left(\frac{T}{10^{4} \mathrm{~K}}\right)^{\frac{-1.35}{2 \alpha-1}}
$$

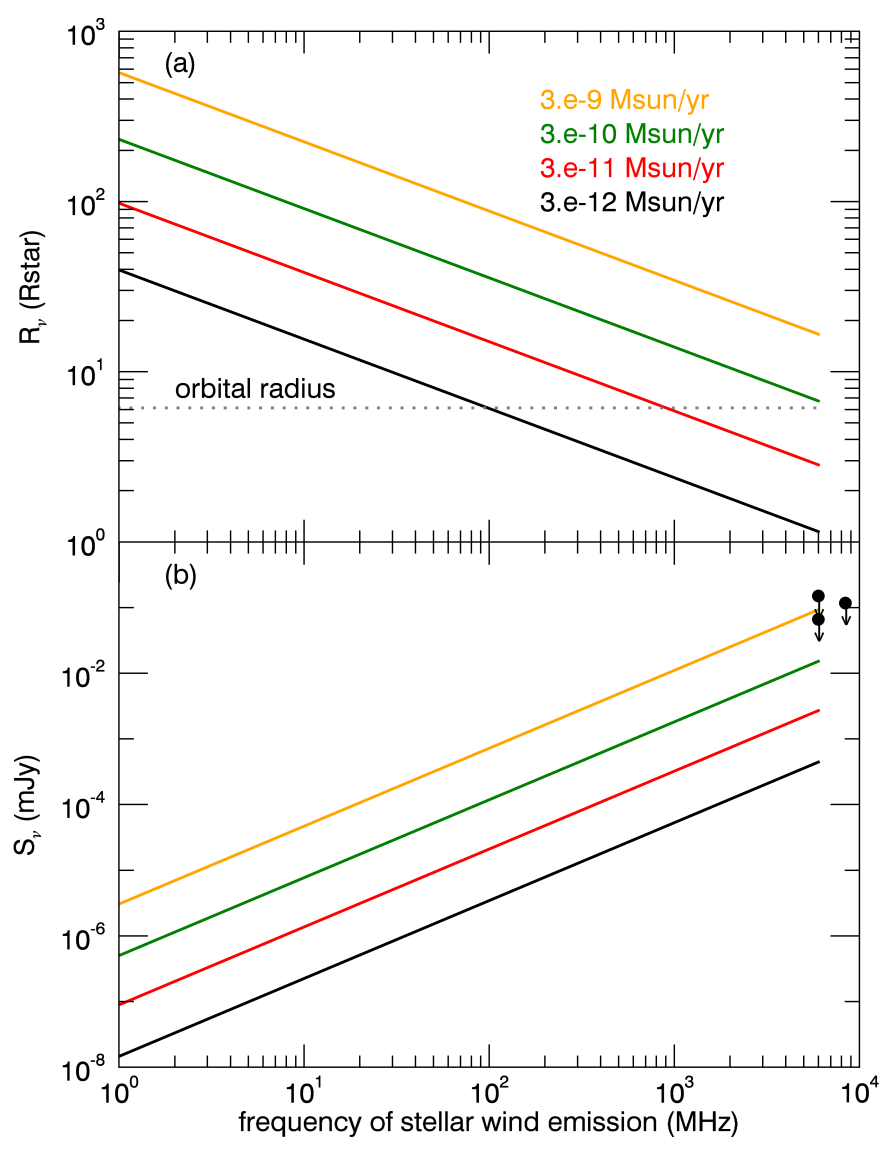

Fig. 4. a) Size of the stellar wind radio-emitting region as a function of frequency for a range of stellar mass-loss rates: $3 \times 10^{-12} M_{\odot} \mathrm{yr}^{-1}$ to $3 \times 10^{-9} M_{\odot} \mathrm{yr}^{-1}$ from bottom to top solid lines. The dotted line indicates the orbital radius of V830 Tau b. b) The same as in a), but for the flux density of the emitting wind, $S_{v}$. Circles are the upper limits for the radio emission of V830 Tau $\mathrm{b}$ as presented by Bower et al. (2016).

For the wind temperature $T$ in Eqs. (9) and (13), we use the temperature adopted at the wind base $\left(10^{6} \mathrm{~K}\right)$ and we use the base density $n_{0}=10^{12} \mathrm{~cm}^{-3}$ adopted in the model described in Sect. 2. To estimate $\alpha$, we compute a power-law fit for regions within $r<20 R_{\star}$, which resulted in $\alpha \simeq 3.08^{3}$. With this value of $\alpha$, the temperature and frequency dependencies of $R_{v}$ become

$R_{v} \propto v^{-0.40} T^{-0.26}$,

which is flatter than an emitting wind that has reached asymptotic speed $\left(R_{v} \propto v^{-0.7} T^{-0.45}\right.$, Guedel 2002).

Figure 4 a shows the size of the radio-emitting region, where the dotted line indicates the orbital radius of V830 Tau b. We also explore the effects of the wind mass-loss rates on the radio emission of the wind. For this estimate, we scale the wind densities of the model presented in Sect. 2, such that the mass-loss rates of our parametric study vary between $10^{-12}$ and $3 \times 10^{-9} M_{\odot} \mathrm{yr}^{-1}$. We remind readers that the model presented in Sect. 2 (our fiducial model) has $\dot{M} \sim 3 \times 10^{-9} M_{\odot} \mathrm{yr}^{-1}$. We note that $R_{v}$ gets smaller for higher frequencies and/or lower mass-loss rates. At $10 \mathrm{MHz}$ and for $\dot{M}$ in the range $\left[3 \times 10^{-12}, 3 \times 10^{-9}\right] M_{\odot} \mathrm{yr}^{-1}$, the planet's orbit is always within $R_{v}$, that is, the planet is embedded in the radio-emitting wind. For $100 \mathrm{MHz}$ and in the same range

\footnotetext{
3 Note that, in the case of a stellar wind that has reached asymptotic wind speed, $\alpha=2$. This reduces, for example, to the case presented in Guedel (2002).
} 
of $\dot{M}, R_{v} \sim 6$ to $90 R_{\star}$ and $r_{\text {orb }} \gtrsim R_{v}$ for $\dot{M} \lesssim 3 \times 10^{-12} M_{\odot} \mathrm{yr}^{-1}$ For $1000 \mathrm{MHz}, r_{\text {orb }}>R_{\nu}$ for $\dot{M} \lesssim 3 \times 10^{-11} M_{\odot} \mathrm{yr}^{-1}$.

Regarding the flux density of the emitting wind, $S_{v}$ increases for higher frequencies and/or higher mass-loss rates. For all the range of $\dot{M}$ we investigated and the range of frequencies $([1,6000] \mathrm{MHz})$, the flux density of the emitting wind never reaches the mJy level and $S_{v} \lesssim 0.1 \mathrm{mJy}$. Given the low values of $S_{v}$ at low frequencies $\left(S_{v} \lesssim 10^{-5} \mathrm{mJy}\right.$ for $\left.v \lesssim 100 \mathrm{MHz}\right)$, detecting radio emission from V830 Tau's wind at these frequencies with present-day technology is not feasible (Sect. 5).

By comparing our radio emission estimates with the observations presented in Bower et al. (2016), we can place constraints on the mass-loss rates of V830 Tau. At certain observing epochs, Bower et al. (2016) detected V830 Tau in a flaring state, while at other observing epochs, only upper limits for radio emission of V830 Tau could be derived, namely, $<0.066$ and $<0.147 \mathrm{mJy}$ at $6 \mathrm{GHz}$ (VLA observations) and $<0.117 \mathrm{mJy}$ at $8.4 \mathrm{GHz}$ (VLBA). These upper limits are shown as circles in Fig. 4b. From that, we infer an upper limit of $\dot{M} \lesssim 3 \times 10^{-9} M_{\odot} \mathrm{yr}^{-1}$ for the mass-loss rate of V830 Tau, which is consistent to the value used in our fiducial model (Sect. 2).

\subsection{Can radio emission from V830 Tau b propagate through the stellar wind?}

The planetary radio emission can only propagate in the stellar wind plasma if the (maximum) frequency of emission $\Omega_{\mathrm{c}}=$ $e B\left(\alpha_{0}\right) /\left(2 \pi m_{\mathrm{e}} c\right)$ is larger than the stellar wind plasma frequency $\omega_{\mathrm{p}}=\left[n_{\mathrm{e}} e^{2} /\left(\pi m_{\mathrm{e}}\right)\right]^{1 / 2}$ everywhere along the propagation path. In these expressions, $n_{\mathrm{e}}$ is the local electron density of the stellar wind ( $n_{\mathrm{e}}=n / 2$ for a fully ionised hydrogen wind), $e$ and $m_{\mathrm{e}}$ are the electron charge and mass, respectively, and $B\left(\alpha_{0}\right)$ is the planetary magnetic field at colatitude $\alpha_{0}$ (half-aperture of the polarcap boundary). In the scenario in which the planetary emission propagates towards wind lower densities, the condition $\Omega_{\mathrm{c}}>\omega_{\mathrm{p}}$ is met when

$B\left(\alpha_{0}\right)>\left(\frac{n_{\mathrm{e}}}{10^{5} \mathrm{~cm}^{-3}}\right)^{1 / 2} \mathrm{G}$

where $n_{\mathrm{e}}$ is taken as the electron density at the planetary orbit. Figure 5 shows the minimum planetary magnetic field intensity of V830 Tau $b$ required for the propagation of planetary radio emission through the wind of the host star, as a function of the stellar wind mass-loss rates.

Mass-loss rates of weak-lined $\mathrm{T}$ Tauri stars have not been observationally constrained. It is expected that these winds are intermediate between those of classical $\mathrm{T}$ Tauri stars with dense winds $\left(\dot{M} \sim 10^{-10}-10^{-8} M_{\odot} \mathrm{yr}^{-1}\right.$, Hartigan et al. 1995 ; Iguchi \& Itoh 2016) and zero-age main sequence stars with less dense winds. Very active, main sequence stars can have massloss rates of $\dot{M} \sim 2 \times 10^{-12} M_{\odot} \mathrm{yr}^{-1}$ (Wood et al. 2005). In our work, we estimate an upper limit for the wind of V830 Tau to be $\dot{M} \lesssim 3 \times 10^{-9} M_{\odot} \mathrm{yr}^{-1}$ (Fig. 4b). Given these values, we speculate that the most probable $\dot{M}$ for the winds of weak-lined T Tauri stars lies in the range $\sim 10^{-12}-10^{-10} M_{\odot} \mathrm{yr}^{-1}$. We use this range of values for the estimates we present next ${ }^{4}$.

For $\dot{M} \sim 10^{-12}-10^{-10} M_{\odot} \mathrm{yr}^{-1}$, the size of the wind-emitting region ranges between $R_{v} \sim 3-15 R_{\star}$ at $275 \mathrm{MHz}$ and between $R_{v} \sim 5-30 R_{\star}$ at $50 \mathrm{MHz}$ (cf. Fig. 4a). At these frequencies, $R_{v}$

\footnotetext{
4 The non-detection of radio emission from stellar winds can be a useful way to observationally constrain mass-loss rates in solar-type stars, which have rarefied winds (e.g. Gaidos et al. 2000; Villadsen et al. 2014; Fichtinger et al. 2017).
}

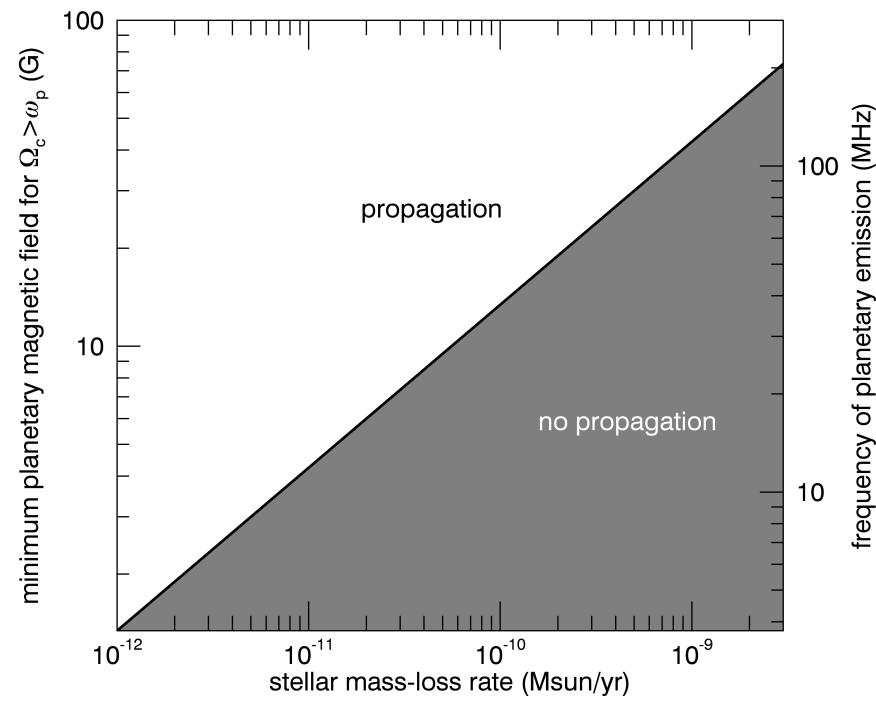

Fig. 5. Minimum planetary magnetic field intensity required for the propagation of planetary radio emission through the wind of the host star (Eq. (15)) as a function of stellar wind mass-loss rate. The right hand side axis shows the corresponding frequency of planetary emission (cyclotron).

is comparable to the planetary orbital radius $\left(6.1 R_{\star}\right)$, implying that V830 Tau b might be embedded in the regions of the stellar wind that are optically thick to radio wavelengths, although not so deeply embedded. Given that the radio flux we computed for the planet $(\sim 6-24 \mathrm{mJy}$, Fig. $3 \mathrm{~b})$ is several orders of magnitude larger that the stellar radio flux $\left(\sim 10^{-6}-10^{-3} \mathrm{mJy}\right.$, Fig. $\left.4 \mathrm{~b}\right)$, it is possible that, even after attenuation, a significant fraction of the planetary radio flux can escape. An accurate radiative transfer calculation of the amount of planetary flux that reaches the observer is left for a more detailed future study.

For the same range of $\dot{M}$, planetary radio emission can propagate through the stellar wind if the planetary magnetic field strength is $\gtrsim 1.3-13 \mathrm{G}$ (Fig. 5). These minimum values encompass Jupiter's magnetic field intensities and appear to be physically reasonable. The equivalent (minimum) frequency of planetary emission is about $\gtrsim 4-40 \mathrm{MHz}$. At a frequency of $\sim 50 \mathrm{MHz}$ (see previous paragraph) the wind is (radio) optically thick out to $\sim 5$ to $30 R_{\star}$ and the planet is not so deeply embedded in the radio-emitting wind. Altogether, these conditions seem very encouraging for planetary radio emission to escape the stellar wind of V830 Tau and be detected at Earth.

\section{Discussion and conclusions}

Based on 3D MHD simulations, we presented here estimates of the radio emission expected for V830 Tau $b$, the youngest detected exoplanet to date. It orbits an active $2 \mathrm{Myr}$-old weak-lined $\mathrm{T}$ Tauri star at a distance of $0.057 \mathrm{au}$, which is about 6.1 stellar radii. At this distance, the environment surrounding this exoplanet is quite harsh. Using the observationally reconstructed stellar magnetic field, we simulated the wind of this star, allowing us to infer the conditions of the external ambient medium surrounding V830 Tau b. With these conditions, we then computed the planetary radio emission, using, as analogy, the radiometric Bode's law derived for the magnetised planets in the solar system. According to this empirical law, there exists a relation between the dissipated power of the stellar wind impacting on the magnetosphere of the planet and the power released 
in the planetary emission. Our model uses simple approximations and should be considered as an initial attempt at calculating the radio flux of V830 Tau $\mathrm{b}$, which is by far currently the best target for detection of exoplanetary radio emission. Detecting V830 Tau b at radio wavelengths would ultimately help us to refine our models.

We showed that, although the frequency of the radio emission is intimately related to the assumed magnetic field of the planet, the radio fluxes are only weakly dependent on that. The estimated flux densities from dissipated magnetic wind energy are on the order of $6 \mathrm{mJy}$, with peaks at $11 \mathrm{mJy}$, for an assumed planetary radius $R_{\mathrm{p}}=R_{\text {jup }}$. Given the youth of V830 Tau b, it is likely that this is a lower limit for the planetary radius. Alternatively, for $R_{\mathrm{p}}=2 R_{\text {jup }}$, the radio fluxes we estimated peak at $44 \mathrm{mJy}$, with average emission of $24 \mathrm{mJy}$. If V830 Tau b were to have a polar magnetic field intensity of $14.3 \mathrm{G}$ (the maximum value of Jupiter's magnetic field), this means that the emission would occur at a frequency of about $28 \mathrm{MHz}$, originating at latitudes of about $34^{\circ}$, where the magnetic field intensity is $\sim 10 \mathrm{G}$. Given that LOFAR sensitivity for a 1-h integration time at $20-40 \mathrm{MHz}$ is $3-30 \mathrm{mJy}$ (Grießmeier et al. 2011), we conclude that V830 Tau b is an excellent target to look for exoplanetary radio emission with LOFAR. Other present-day instruments that have the potential to detect such radio fluxes in the lowfrequency range would be the upgraded UTR-2 (Ryabov et al. 2010) and GMRT (Sirothia et al. 2014). In the near future, the Square Kilometre Array (SKA)-low array system is expected to outperform LOFAR in terms of sensitivity limits for low frequency ranges (Zarka et al. 2015) and it would be an ideal tool for detecting exoplanets at radio wavelengths.

In Vidotto et al. (2012, 2015), the radio flux densities of several hot Jupiters (namely: $\tau$ Boo b, HD 46375b, HD 73256b, HD 102195b, HD 130322b, HD 179949b, and HD 189733b) were computed using the same method as here, that is, one in which the data-driven 3D MHD simulations provide the stellar wind characteristics used in the radio flux computations. Compared to the radio flux densities derived in those past works $(0.02$ to $1 \mathrm{mJy}), \mathrm{V} 830 \mathrm{Tau} \mathrm{b}$ presents the best prospect for detecting radio emission (in spite of it not being the closest among the studied systems). This is because V830 Tau is an active star with a large-scale surface magnetic field intensity reaching $700 \mathrm{G}$ (Donati et al. 2015, 2016), up to two orders of magnitude larger than the magnetism of the stars studied in Vidotto et al. (2015). The latter are main-sequence solar-type stars, which are significantly older than V830 Tau. In their youth, stars are more active and their magnetism more intense (Vidotto et al. 2014a).

In addition to the variability of radio emission seen along one planetary year (Figs. 2 and 3, see also Fares et al. 2010; Vidotto et al. 2010b, 2012, 2015), variation within a few years timescale is also expected due to the evolution of the host star magnetism. This means that, within a few years, the conditions of the stellar wind surrounding the planet are likely to change, causing also changes in exoplanetary radio emission. This could be challenging for radio detection, which will likely require some monitoring of the system.

The star itself may also contribute at radio frequencies (and potentially through flares that could make the planet modulation harder to detect). We computed thermal radio emission generated in the high density regions of the stellar wind. Comparing our estimated values with the non-detections reported in Bower et al. (2016), we were able to place a constraint in the mass-loss rate of V830 Tau: $\dot{M} \lesssim 3 \times 10^{-9} M_{\odot} \mathrm{yr}^{-1}$. We argued that the most likely values of $\dot{M}$ lie between $\sim 10^{-12}$ and $10^{-10} M_{\odot} \mathrm{yr}^{-1}$. With these values, the radio-emitting wind extends to distances of $\sim 3$ to $30 R_{\star}$ at frequencies of 50 to $275 \mathrm{MHz}$. This implies that the orbit of V830 Tau b (at $6.1 R_{\star}$ ) might sit inside the radio-emitting wind, but does not seem to be deeply embedded. Given that the radio fluxes of the planet are estimated to be $\sim 3$ to 7 orders of magnitude larger than the radio flux of the stellar wind, it is possible that even after undergoing absorption by the stellar wind, a significant fraction of the planetary radio emission can escape the optically (radio) thick wind and reach the observer. Altogether, the V830 Tau system is a very encouraging system for conducting radio observations from both the perspective of radio emission from the planet as well as from the host star's wind. Equally important, the lack of detection of emission at radio wavelengths can place important constraints on the characteristics of the yet unobserved winds from weak-lined T Tauri stars.

Finally, exoplanetary radio emission may offer a new avenue for detecting young hot giants. The recent discovery of three of them (V830 Tau b, K2-33b, and TAP26b) may, in fact, indicate that these planets are more frequent than their equivalents around mature Sun-like stars. However, the extreme activity levels of their hosts prevents the use of traditional planet-search techniques. Radio searches can thus boost the findings of young hot Jupiters. It should also give us the chance to probe their magnetic fields from the detected radio frequency, and to simultaneously study stellar winds and star-planet interactions.

Acknowledgements. This work used the BATS-R-US tools developed at the University of Michigan Center for Space Environment Modeling and made available through the NASA Community Coordinated Modeling Center. This work was supported by a grant from the Swiss National Supercomputing Centre (CSCS) under project ID s516. J.F.D. thanks the IDEX initiative of Université Fédérale Toulouse Midi-Pyrénées for awarding a Chaire d'Attractivité in the framework of which the study of V830 Tau was carried out. The authors would like to thank Manuel Guedel and the anonymous referee for their constructive comments, which greatly improved this manuscript.

\section{References}

Bagenal, F. 1992, Ann. Rev. Earth Planet. Sci., 20, 289

Bastian, T. S., Dulk, G. A., \& Leblanc, Y. 2000, ApJ, 545, 1058

Bower, G. C., Loinard, L., Dzib, S., et al. 2016, ApJ, 830, 107

Cravens, T. E. 2004, Physics of Solar System Plasmas (Cambridge, UK: Cambridge University Press)

David, T. J., Hillenbrand, L. A., Petigura, E. A., et al. 2016, Nature, 534, 658 do Nascimento, Jr., J.-D., Vidotto, A. A., Petit, P., et al. 2016, ApJ, 820, L15 Donati, J.-F., Hébrard, E., Hussain, G. A. J., et al. 2015, MNRAS, 453, 3706 Donati, J. F., Moutou, C., Malo, L., et al. 2016, Nature, 534, 662 Fares, R., Donati, J., Moutou, C., et al. 2010, MNRAS, 406, 409

Farrell, W. M., Desch, M. D., \& Zarka, P. 1999, J. Geophys. Res., 104, 14025 Fichtinger, B., Güdel, M., Mutel, R. L., et al. 2017, A\&A, 599, A127

Gaidos, E. J., Guedel, M., \& Blake, G. A. 2000, Geophys. Res. Lett., 27, 501

Grießmeier, J.-M., Motschmann, U., Mann, G., \& Rucker, H. O. 2005, A\&A, 437, 717

Grießmeier, J.-M., Preusse, S., Khodachenko, M., et al. 2007, Planet. Space Sci., 55,618

Grießmeier, J.-M., Zarka, P., \& Girard, J. N. 2011, Radio Science, 46, 0

Grießmeier, J.-M., Tabataba-Vakili, F., Stadelmann, A., Grenfell, J. L., \& Atri, D. 2016, A\&A, 587, A159

Guedel, M. 2002, ARA\&A, 40, 217

Hallinan, G., Sirothia, S. K., Antonova, A., et al. 2013, ApJ, 762, 34

Hartigan, P., Edwards, S., \& Ghandour, L. 1995, ApJ, 452, 736

Hess, S. L. G., \& Zarka, P. 2011, A\&A, 531, A29

Hill, T. W. 2001, J. Geophys. Res., 106, 8101

Iguchi, N., \& Itoh, Y. 2016, Res. Astron. Astrophys., 16, 010

Jardine, M., \& Cameron, A. C. 2008, A\&A, 490, 843

Johnstone, C. P., Guedel, M., Brott, I., \& Lüftinger, T. 2015, A\&A, 577, A28 Kislyakova, K. G., Holmström, M., Lammer, H., Odert, P., \& Khodachenko, M. L. 2014, Science, 346, 981

Lazio, T. J. W., \& Farrell, W. M. 2007, ApJ, 668, 1182

Lazio, T. J. W., Farrell, W. M., Dietrick, J., et al. 2004, ApJ, 612, 511 
Lecavelier des Etangs, A., Sirothia, S. K., Gopal-Krishna, \& Zarka, P. 2013, A\&A, 552, A65

Lim, J., \& White, S. M. 1996, ApJ, 462, L91

Lundin, R., Lammer, H., \& Ribas, I. 2007, Space Sci. Rev., 129, 245

Milan, S. E., Hutchinson, J., Boakes, P. D., \& Hubert, B. 2009, Annales Geophysicae, 27, 2913

Nichols, J. D., \& Milan, S. E. 2016, MNRAS, 461, 2353

Panagia, N., \& Felli, M. 1975, A\&A, 39,

Powell, K. G., Roe, P. L., Linde, T. J., Gombosi, T. I., \& de Zeeuw, D. L. 1999, J. Chem. Phys., 154, 284

Preusse, S., Kopp, A., Büchner, J., \& Motschmann, U. 2005, A\&A, 434, 1191

Ryabov, V. B., Zarka, P., \& Ryabov, B. P. 2004, Planet. Space Sci., 52, 1479

Ryabov, V. B., Vavriv, D. M., Zarka, P., et al. 2010, A\&A, 510, A16

Santerne, A., Hébrard, G., Lillo-Box, J., et al. 2016, ApJ, 824, 55

Saur, J., Grambusch, T., Duling, S., Neubauer, F. M., \& Simon, S. 2013, A\&A, 552, A 119

See, V., Jardine, M., Vidotto, A. A., et al. 2014, A\&A, 570, A99

Shkolnik, E., Bohlender, D. A., Walker, G. A. H., \& Collier Cameron, A. 2008, ApJ, 676, 628

Sirothia, S. K., Lecavelier des Etangs, A., Gopal-Krishna, Kantharia, N. G., \& Ishwar-Chandra, C. H. 2014, A\&A, 562, A108

Siscoe, G. L., \& Chen, C.-K. 1975, J. Geophys. Res., 80, 4675

Strugarek, A., Brun, A. S., Matt, S. P., \& Réville, V. 2015, ApJ, 815, 111

Tarduno, J. A., Cottrell, R. D., Watkeys, M. K., et al. 2010, Science, 327, 1238

Tóth, G., van der Holst, B., Sokolov, I. V., et al. 2012, J. Comput. Phys., 231, 870

Vidotto, A. A., Opher, M., Jatenco-Pereira, V., \& Gombosi, T. I. 2009, ApJ, 699, 441
Vidotto, A. A., Jardine, M., \& Helling, C. 2010a, ApJ, 722, L168

Vidotto, A. A., Opher, M., Jatenco-Pereira, V., \& Gombosi, T. I. 2010b, ApJ, 720,1262

Vidotto, A. A., Jardine, M., \& Helling, C. 2011, MNRAS, 414, 1573

Vidotto, A. A., Fares, R., Jardine, M., et al. 2012, MNRAS, 423, 3285

Vidotto, A. A., Gregory, S. G., Jardine, M., et al. 2014a, MNRAS, 441, 2361

Vidotto, A. A., Jardine, M., Morin, J., et al. 2014b, MNRAS, 438, 1162

Vidotto, A. A., Fares, R., Jardine, M., Moutou, C., \& Donati, J.-F. 2015, MNRAS, 449, 4117

Villadsen, J., Hallinan, G., Bourke, S., Guedel, M., \& Rupen, M. 2014, ApJ, 788, 112

Weber, E. J., \& Davis, L. J. 1967, ApJ, 148, 217

Wood, B. E. 2004, Liv. Rev. Sol. Phys., 1, 2

Wood, B. E., Müller, H.-R., Zank, G. P., Linsky, J. L., \& Redfield, S. 2005, ApJ, $628, \mathrm{~L} 143$

Wright, A. E., \& Barlow, M. J. 1975, MNRAS, 170, 41

Yu, L., Donati, J.-F., Hébrard, E. M., et al. 2017, MNRAS, 467, 1342

Zarka, P. 2007, Planet. Space Sci., 55, 598

Zarka, P. 2010, in Pathways Towards Habitable Planets, eds. V. Coudé du Foresto, D. M. Gelino, \& I. Ribas, ASP Conf. Ser., 430, 175

Zarka, P., Treumann, R. A., Ryabov, B. P., \& Ryabov, V. B. 2001, Ap\&SS, 277, 293

Zarka, P., Cecconi, B., \& Kurth, W. S. 2004, J. Geophys. Res. (Space Physics), 109,9

Zarka, P., Lazio, J., \& Hallinan, G. 2015, Advancing Astrophysics with the Square Kilometre Array (AASKA14), 120

Zuluaga, J. I., Bustamante, S., Cuartas, P. A., \& Hoyos, J. H. 2013, ApJ, 770, 23 


\section{Appendix A: Detailed derivation of planetary radio flux}

In this work, we assume that radio emission arises near the colatitude $\alpha_{0}$ of the open-closed magnetic field line boundary; we refer to the region of open field lines as the polar cap ${ }^{5}$. We note that the colatitudes of the polar cap boundary and that of the auroral ring might not match exactly (Siscoe \& Chen 1975; Hill 2001), so this is to be considered as a first-order approximation. The half-aperture of the polar cap boundary $\alpha_{0}$ can be related to the size of the planet magnetosphere as (Siscoe \& Chen 1975; Tarduno et al. 2010; Vidotto et al. 2011; Zuluaga et al. 2013)

$\alpha_{0}=\arcsin \left[\left(R_{\mathrm{p}} / r_{\mathrm{M}}\right)^{1 / 2}\right]$.

The (dipolar) planetary magnetic field at this colatitude $\alpha_{0}$ is

$B\left(\alpha_{0}\right)=\frac{B_{\mathrm{p}}}{2}\left(1+3 \cos ^{2} \alpha_{0}\right)^{1 / 2}$,

which corresponds to a maximum electron cyclotron frequency

$f_{\mathrm{c}}=\frac{e B\left(\alpha_{0}\right)}{2 \pi m_{\mathrm{e}} c}$,

where $m_{\mathrm{e}}$ and $e$ are the electron mass and charge, and $c$ is the speed of light (Fig. A.1). Here, we assume that the emission bandwidth $\Delta f$ is approximately the cyclotron frequency (Grießmeier et al. 2007):

$\Delta f=\frac{e B\left(\alpha_{0}\right)}{2 \pi m_{\mathrm{e}} c}=2.8\left(\frac{B\left(\alpha_{0}\right)}{1 \mathrm{G}}\right) \mathrm{MHz}$.

The radio flux is related to the radio power as

$\phi_{\text {radio }}=\frac{P_{\text {radio }}}{d^{2} \omega \Delta f}$,

where $d$ is the distance to the system and

$$
\begin{aligned}
\omega & =2 \times \int_{\alpha_{0}-\delta \alpha / 2}^{\alpha_{0}+\delta \alpha / 2} \sin \alpha \mathrm{d} \alpha \mathrm{d} \varphi \\
& =2 \times 2 \pi\left[\cos \left(\alpha_{0}-\delta \alpha / 2\right)-\cos \left(\alpha_{0}+\delta \alpha / 2\right)\right]
\end{aligned}
$$

is the solid angle of the hollow cone where emission is arising (the factor of two was included in order to account for emission coming from both Northern and Southern hemispheres). The thickness of the hollow cone is assumed to be $\delta \alpha=17.5^{\circ}$ (Zarka et al. 2004).

\section{A.1. Flux released in the dissipation of the stellar wind kinetic power}

The dissipated kinetic power of the impacting wind on the planet is approximated as the ram pressure of the wind $\rho(\Delta u)^{2}$ impacting in the magnetospheric cross section $\pi r_{\mathrm{M}}^{2}$, at a (relative) velocity $\Delta u$ (Zarka 2007)

$P_{\mathrm{k}} \simeq \rho(\Delta u)^{3} \pi r_{\mathrm{M}}^{2}$

\footnotetext{
5 Although we refer to $\alpha_{0}$ as the "polar cap boundary", we note that, due to the harsh conditions imposed by the stellar wind environment, the magnetospheric sizes might be small and, therefore, the polar cap might occupy a large area of the surface of the star (i.e. not only immediately around the poles, as happens for instance with the polar cap area of the Earth).
}

The flux that would be emitted when the kinetic power of the wind gets dissipated in the magnetospheric cross section of the planet - if the radio-magnetic scaling law is effective - can be written as

$\phi_{\text {radio,kin }}=\frac{P_{\text {radio }}}{d^{2} \omega \Delta f}=\frac{\eta_{\mathrm{k}} P_{\mathrm{k}}}{d^{2} \omega \Delta f}=\frac{\eta_{\mathrm{k}} \rho(\Delta u)^{3} \pi r_{\mathrm{M}}^{2}}{d^{2} \omega \Delta f}$,

where we used Eqs. (A.5) and (A.7). Using Eqs. (A.1), (A.2), (A.4), and (A.6), the previous expression becomes

$\phi_{\text {radio,kin }}=\frac{\eta_{\mathrm{k}} \rho(\Delta u)^{3} R_{\mathrm{p}}^{2}\left[\cos \left(\alpha_{0}-\delta \alpha / 2\right)-\cos \left(\alpha_{0}+\delta \alpha / 2\right)\right]^{-1}}{d^{2} 2 e /\left[2 \pi m_{\mathrm{e}} c\right] B_{\mathrm{p}}\left(1+3 \cos ^{2} \alpha_{0}\right)^{1 / 2} \sin ^{4} \alpha_{0}}$.

We note that $\alpha_{0}$ correlates to $B_{\mathrm{p}}$ through Eqs. (A.1) and (4) as

$B_{\mathrm{p}}=\frac{2 \sqrt{8 \pi p_{\mathrm{tot}}}}{\sin ^{6} \alpha_{0}}$

where $p_{\text {tot }}=\left(\rho \Delta u^{2}+p+B^{2} / 8 \pi\right)$ is the total pressure of the ambient medium external to the planet. Therefore, Eq. (A.9) becomes

$\phi_{\text {radio,kin }}=$

$$
\begin{aligned}
& \frac{\eta_{\mathrm{k}} \rho(\Delta u)^{3} R_{\mathrm{p}}^{2} \sin ^{2} \alpha_{0}\left[\cos \left(\alpha_{0}-\delta \alpha / 2\right)-\cos \left(\alpha_{0}+\delta \alpha / 2\right)\right]^{-1}}{d^{2} 4 e /\left[2 \pi m_{\mathrm{e}} c\right] \sqrt{8 \pi p_{\mathrm{tot}}}\left(1+3 \cos ^{2} \alpha_{0}\right)^{1 / 2}} \\
= & \eta_{\mathrm{k}} \frac{2 \pi m_{\mathrm{e}} c}{4 e} \frac{R_{\mathrm{p}}^{2}}{d^{2}} \frac{\rho(\Delta u)^{3}}{\sqrt{8 \pi p_{\text {tot }}}} f\left(\alpha_{0}\right)
\end{aligned}
$$

with

$f\left(\alpha_{0}\right)=\frac{\sin ^{2} \alpha_{0}}{\left[\cos \left(\alpha_{0}-\delta \alpha / 2\right)-\cos \left(\alpha_{0}+\delta \alpha / 2\right)\right]\left(1+3 \cos ^{2} \alpha_{0}\right)^{1 / 2}}$.

We note that the planet magnetic field $B_{\mathrm{p}}$ is no longer explicit in Eq. (A.11); the dependence of the $\phi_{\text {radio,kin with } B_{\mathrm{p}} \text { is hidden }}$ in $\alpha_{0}$ (Eq. (A.10)) and, consequently, in $f\left(\alpha_{0}\right)$. Figure A. 1 shows the variation of $f$ as a function of a given co-latitude of the polarcap boundary $\alpha_{0}$. As can be seen, this function varies between 0 and 3.3 for any aperture angle of the polar-cap boundary.

If we then group all the constants of Eq. (A.11) into

$\eta_{\mathrm{k}}^{\prime}=\eta_{\mathrm{k}} \frac{2 \pi m_{\mathrm{e}} c}{4 e \sqrt{8 \pi}} \simeq \eta_{\mathrm{k}} 1.8 \times 10^{-8}$ [cgs units]

the radio flux due to the impacting wind (kinetic power) simplifies to

$\phi_{\text {radio,kin }}=\eta_{\mathrm{k}}^{\prime} \frac{R_{\mathrm{p}}^{2}}{d^{2}} \frac{\rho(\Delta u)^{3}}{p_{\text {tot }}^{1 / 2}} f\left(\alpha_{0}\right)$,

which is a function of the angular size of the planet $\left(R_{\mathrm{p}} / d\right)$, the properties of the ambient medium surrounding the planet (i.e. the stellar wind), and $f(\alpha)$. 


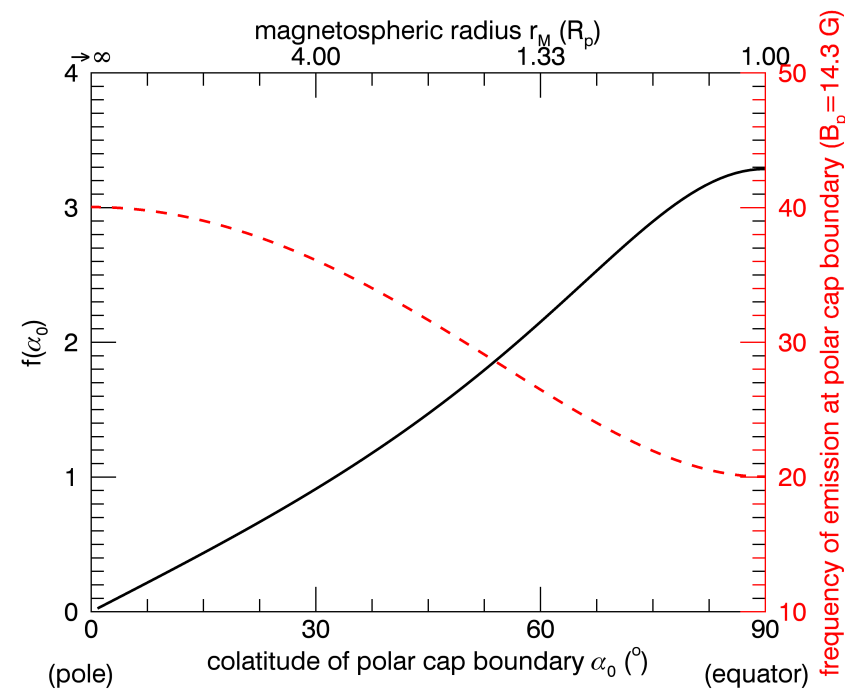

Fig. A.1. Black solid curve: variation of $f$ (Eq. (A.12)) as a function of the planetary co-latitude of the polar-cap boundary $\alpha_{0}$. The top axis indicates the (non-linear) conversion from $\alpha_{0}$ to a normalised magnetospheric size following Eq. (A.1). Red dashed curve: the emission frequency at the polar-cap boundary (Eq. (A.3), right axis) for a magnetic field intensity at the pole of $14.3 \mathrm{G}$.

\section{A.2. Flux released in the dissipation of the stellar wind Poynting flux}

The magnetic power $P_{B}$ can be estimated as the Poynting flux of the stellar wind impacting on the planetary magnetospheric cross-section $S$ (Zarka 2007)

$P_{B}=\int c \frac{\boldsymbol{E} \times \boldsymbol{B}}{4 \pi} \mathrm{d} \boldsymbol{S} \simeq \frac{B_{\perp}^{2}}{4 \pi}(\Delta u) \pi r_{\mathrm{M}}^{2}$,

where the electric field is $\boldsymbol{E}=-\Delta \boldsymbol{u} \times \boldsymbol{B} / c$ and $B_{\perp}$ is the perpendicular component of the stellar magnetic field to the velocity of the wind particles at the planet's referencial frame. It can be written as

$B_{\perp}^{2}=B^{2}-B_{\|}^{2}=B^{2}-\left(\frac{\boldsymbol{B} \cdot \Delta \boldsymbol{u}}{|\Delta \boldsymbol{u}|}\right)^{2}$.

The power dissipated in interaction is often ascribed as a fraction $\epsilon$ of Eq. (A.15), with $\epsilon \sim 0.1$ to 0.2 (Zarka 2007). However, this efficiency factor is often included in the radiometric Bode's law by means of the $\eta_{B}$ parameter, such that $P_{\text {radio }}=\eta_{B} P_{\mathrm{B}}$, with $\eta_{B}=2 \times 10^{-3}$ and $P_{\mathrm{B}}$ as in Eq. (A.15).

The flux that is emitted with the dissipation of wind magnetic power can be written as

$\phi_{\text {radio,mag }}=\frac{P_{\text {radio }}}{d^{2} \omega \Delta f}=\frac{\eta_{B} P_{\mathrm{B}}}{d^{2} \omega \Delta f}=\frac{\eta_{B} B_{\perp}^{2}(\Delta u) \pi r_{\mathrm{M}}^{2}}{4 \pi d^{2} \omega \Delta f}$,

where we used Eqs. (A.15) and (A.5). Using Eqs. (A.1), (A.2), (A.4), (A.6), and (A.10), the previous expression becomes

$$
\begin{aligned}
& \phi_{\text {radio,mag }}= \\
& \frac{\eta_{B} B_{\perp}^{2}(\Delta u)\left[\cos \left(\alpha_{0}-\delta \alpha / 2\right)-\cos \left(\alpha_{0}+\delta \alpha / 2\right)\right]^{-1} R_{\mathrm{p}}^{2} \sin ^{2} \alpha_{0}}{4 \pi d^{2} 4 e /\left[2 \pi m_{\mathrm{e}} c\right] \sqrt{8 \pi p_{\mathrm{tot}}}\left(1+3 \cos ^{2} \alpha_{0}\right)^{1 / 2}} \\
& \quad=\eta_{B} \frac{2 \pi m_{\mathrm{e}} c}{4 e} \frac{R_{\mathrm{p}}^{2}}{d^{2}} \frac{B_{\perp}^{2}(\Delta u)}{4 \pi \sqrt{8 \pi p_{\text {tot }}}} f\left(\alpha_{0}\right) .
\end{aligned}
$$

The magnitude of the planetary magnetic field is again embedded in $f\left(\alpha_{0}\right)$ (cf. Fig. A.1). If we then group all the constants of Eq. (A.18) into

$\eta_{B}^{\prime}=\eta_{B} \frac{2 \pi m_{\mathrm{e}} c}{4 e \sqrt{8 \pi}} \frac{1}{4 \pi} \simeq \eta_{B} 1.4 \times 10^{-9}$ [cgs units],

the radio flux due to the impacting wind (magnetic power) simplifies to

$\phi_{\text {radio,mag }}=\eta_{B}^{\prime} \frac{R_{\mathrm{p}}^{2}}{d^{2}} \frac{B_{\perp}^{2}(\Delta u)}{p_{\text {tot }}^{1 / 2}} f\left(\alpha_{0}\right)$,

which is a function of the angular size of the planet $\left(R_{\mathrm{p}} / d\right)$, the properties of the ambient medium surrounding the planet (i.e. the stellar wind), and $f(\alpha)$.

\section{Appendix B: Effects of wind base density in our predicted radio emission}

In our simulations of V830 Tau wind, we noted in Sect. 2 that the base density $n_{0}$ of the wind is a free parameter that affects the computed mass-loss rates and that, with our choice of $n_{0}$, our computed mass-loss rates were likely near an upper limit of the expected mass-loss rates for weak-lined T Tauri stars. Next, we verify how the radio fluxes estimated in this paper would have changed if the wind densities were to decrease by one to two orders of magnitude, bringing down the wind mass-loss rates of V830 Tau to $\sim 10^{-11}-10^{-10} M_{\odot} \mathrm{yr}^{-1}$.

With the wind densities assumed in this paper, the planet orbit changes from a magnetically-dominated region to a rampressure dominated region, as shown in Fig. 1. A decrease in wind density by a factor of 10-100 would imply that the planet's orbit would be completely inside the magnetically-dominated region (sub-Alfvénic). We remind readers also that, even if a given stellar wind is not dominated by the magnetic pressure, $\phi_{\text {radio,mag }}$ can still dominate over $\phi_{\text {radio,kin }}$ because of the $3200 \pi^{2}$ factor in Eq. (8).

To simplify our following estimates, we study two limiting cases: (i) one in which the ram pressure dominates the stellar wind total pressure (similar to the conditions surrounding the Earth) and (ii) one in which the magnetic pressure is the dominating one (likely to be the condition surrounding V830 Tau b). In case (i), we have that $p_{\text {tot }} \rightarrow \rho(\Delta u)^{2}$, and Eqs. (A.14) and (A.20) become

$\phi_{\text {radio,kin }} \rightarrow \eta_{\mathrm{k}}^{\prime} \frac{R_{\mathrm{p}}^{2}}{d^{2}} \rho^{1 / 2}(\Delta u)^{2} f\left(\alpha_{0}\right) \quad$ [ram-pressure dominated]

and

$\phi_{\text {radio,mag }} \rightarrow \eta_{B}^{\prime} \frac{R_{\mathrm{p}}^{2}}{d^{2}} \frac{B_{\perp}^{2}}{\rho^{1 / 2}} f\left(\alpha_{0}\right) \quad$ [ram-pressure dominated].

That is, $\phi_{\text {radio,kin }}$ becomes proportional to the square root of the stellar wind density, and $\phi_{\text {radio,mag }}$ becomes inversely proportional to $\rho^{1 / 2}$. In case (ii), where the magnetic pressure of the stellar wind dominates the total pressure $\left(p_{\text {tot }} \rightarrow B^{2} /(8 \pi)\right)$, Eqs. (A.14) and (A.20) become

$\phi_{\text {radio,kin }} \rightarrow \eta_{\mathrm{k}}^{\prime} \frac{R_{\mathrm{p}}^{2}}{d^{2}} \sqrt{8 \pi} \frac{\rho(\Delta u)^{3}}{B} f\left(\alpha_{0}\right)$ [magnetic-pressure dominated], 
A. A. Vidotto and J.-F. Donati: Predicting radio emission from V830 Tau b and its host star

and

$\phi_{\text {radio,mag }} \rightarrow \eta_{B}^{\prime} \frac{R_{\mathrm{p}}^{2}}{d^{2}} \sqrt{8 \pi} B \Delta u f\left(\alpha_{0}\right)$

[magnetic-pressure dominated].

(B.4)

That is, $\phi_{\text {radio,kin }}$ becomes linearly proportional to the stellar wind density, while $\phi_{\text {radio,mag }}$ becomes independent of that.

Therefore, for planets orbiting farther out (in ram-pressure dominated regions), the stellar wind densities can either increase or decrease radio emission depending on whether it is the kinetic or magnetic flux of the impacting wind that is converted into radio power. On the other hand, for close-in planets orbiting around highly magnetised stars (in the magnetic-pressure dominated region), the radio flux becomes roughly independent of the density, being mainly affected by the local conditions of the stellar magnetic field and the relative velocity of the planet through the stellar wind (Eq. (B.4)). Since the latter condition is the most likely for V830 Tau b, we conclude that the stellar wind density, an unknown in our numerical simulations, plays a minor role in our estimates of planetary radio fluxes. 